\title{
A Mean Field Analysis of Pairing in Asymmetric Fermi Systems at Finite Temperature
}

\author{
Rishi Sharma1, * and Sanjay Reddy, \\ ${ }^{1}$ Theoretical Division, Los Alamos National Laboratory, \\ Los Alamos, NM 87545, USA.
}

(Dated: October 31, 2018)

\begin{abstract}
We study the phase diagram of a two component Fermi system with a weak attractive interaction. Our analysis includes the leading order Hartree energy shifts and pairing correlations at finite temperature and chemical potential difference between the two fermion species. We show that in an asymmetric system, the Hartree shift to the single particle energies are important for the phase competition between normal and superfluid phase and can change the phase transition curve qualitatively. At large asymmetry we find that a novel but somewhat fragile superfluid state can be favored due to finite temperature effects. We also investigate the transition between the normal phase and an inhomogeneous superfluid phase to study how gradient instabilities evolve with temperature and asymmetry. Finally, we adopt our analysis to study the density profiles of similar asymmetric Fermi systems that are being observed in cold atom experiments.
\end{abstract}

PACS numbers: 74.25.Dw,74.20.Fg

\section{INTRODUCTION}

The superfluid nature of the ground state of symmetric two-component Fermi systems, where pairing occurs between equal densities of the two species, has been well established, both on theoretical and experimental grounds, since the pioneering work of Bardeen, Cooper and Schrieffer (BCS) fifty years ago 11. However, the phase structure of asymmetric Fermi systems still remains unclear. In particular, theoretical work suggests several novel and competing superfluid phases may be possible. These include: (i) the Ferrel, Fulde, Larkin, Ovchinnikov (FFLO) phase, which is an inhomogeneous superfluid phase with a spatially varying superfluid order parameter [2, 3]; (ii) the breached-pair or gapless superfluid phase, which is a homogeneous superfluid phase containing gapless fermionic excitations even at zero temperature [4]; and (iii) phases with broken rotational symmetry where pairing is facilitated by a deformation of the Fermi surface [5, 6]. Quantum Monte Carlo (QMC) calculations have been used to calculate the thermodynamic properties of a symmetric system at zero temperature 7] for arbitrary interaction strength. The authors of [8] used these results to deduce the presence of a splitting point in the phase diagram of asymmetric systems in the strong interaction regime, where a gapped superfluid phase, a gapless superfluid phase and an inhomogeneous superfluid phase coexist. Their analysis suggests that with the interaction strength tuned to a value such that the $s$-wave scattering length between the two species is infinite, on increasing the difference in the chemical potentials of the two species the system undergoes a first order transition from a homogeneous gapped superfluid phase to an inhomogeneous phase, before eventually transiting to the normal phase.

To investigate the possible existence of these phases, cold atom experiments are now exploring the thermodynamic and linear response properties of asymmetric Fermi systems with attractive interactions 9, 10, 11, 12, 13. These experiments which trap and cool two hyperfine states of ${ }^{6} \mathrm{Li}$ atoms, have unprecedented control over the sample. They can: (i) magnetically tune the interaction strength between the two hyperfine states through Feshbach resonances; (ii) control the population asymmetry by loading different

*Electronic address: rishi@lanl.gov

†Electronic address: reddy@lanl.gov 
numbers of atoms; and (iii) vary the temperature. In the strongly interacting regime, where the two-body scattering length is large, these experiments have already observed how the superfluid properties change with number asymmetry and temperature. Experimental measurements of the density profiles and the response to radio frequency probes seem to indicate that these novel phases are not realized in cold atom traps. Instead one finds strong indications of a first-order phase transition between a superfluid state with zero number asymmetry and a normal state with a large asymmetry [9, 12].

The absence of intervening novel superfluid phases in cold atom experiments is intriguing. These phases may still exist at very low temperature and in the weak coupling regime. To address if these phases can be realized in experiments in the future, we investigate the phase structure of asymmetric Fermi systems at finite temperature and establish the parameter region where interesting new phases of superfluidity can be realized. Our study differs from similar investigations reported in Ref. [14, 15] in two ways. (For a discussion of the phase structure of asymmetric systems at finite temperature in condensed matter systems, see [16, 17, 18, 19].) First, we restrict our analysis to the weak coupling region and perform a self-consistent calculation of the thermodynamic properties of both the normal and the superfluid phases within the purview of mean field theory (Hartree approximation). One drawback of this approach is that we neglect particle-hole screening which is important in the gap equation at weak coupling 20, 21]. This screening is expected to reduce the gap by a factor $\simeq 2.2$. In the conclusions (section IV) we will return to a discussion of how this suppression may affect our final answers. Second, we establish the region in temperature and number asymmetry where the homogeneous states are unstable with respect to small amplitude perturbations.

Although our analysis is strictly valid only in the weak coupling regime, unlike earlier work, we properly account for the single particle energy shifts - or Hartree corrections - that are present both in the normal and superfluid state, in a self-consistent field theoretic approach. We find that these corrections can change the location and shape of the phase boundaries on the phase diagram of the two component Fermi gas. We also find that a new gapless, finite temperature superfluid state is favored over the normal state at temperatures above the critical temperature for the first-order transition. In a narrow window of chemical potential asymmetry, with increasing temperature there is first a first-order transition from the superfluid to the normal phase and then at higher temperature a second-order transition to a weakly superfluid state which exists in limited temperature interval. In this state, finite temperature effects that smear the Fermi surface, facilitate pairing. We will refer to this homogeneous phase as the "fragile" superfluid and it has lower energy than the normal state but is unstable with respect to gradient instabilities.

To study these instabilities we expand the free energy in a Ginzburg-Landau series about the solution of the gap equation, and calculate the coefficient of the quadratic term in the order parameter as a function of its Fourier mode index. We ask the question whether this coefficient is negative for some Fourier mode(s), indicating a gradient instability. The simplest case is an expansion about the normal phase with a zero background value for the difermion condensate. In the presence of a small position dependent condensate

$$
\eta(\mathbf{r})=\int \mathbb{d}^{3} \mathbf{k} e^{i \mathbf{k} \cdot \mathbf{r}} \eta(\mathbf{k}),
$$

we write the change in the free energy as,

$$
\int \vec{d}^{3} \mathbf{k}(\alpha+f(|\mathbf{k}|)) \eta(\mathbf{k}) \eta(-\mathbf{k})+\mathcal{O}\left(\eta^{4}\right),
$$

where we have split the terms in a way that $f(|\mathbf{k}|)$ is zero for $\mathbf{k}=\mathbf{0} . \quad \alpha<0$ points to an instability towards the formation of a homogeneous condensate while $f(|\mathbf{k}|)<0$ points to an instability towards an inhomogeneous modulation of the condensate. We find that there is a window of temperatures and chemical potential splitting where the inhomogeneous superfluid phases may be favored.

The plan of the paper is as follows. We begin by writing down the lagrangian, and reviewing how to include Hartree corrections to the calculation of the gap parameter $\Delta$ and the free energy $\Omega$ of the system in section II. We then proceed with the calculation of $\Delta$ and $\Omega$ in section $\Pi$ which will let us find the phase boundary between the superfluid and the normal phase. We first consider homogeneous phases, meaning $\Delta$ independent of position, and follow with a discussion of inhomogeneous phases. We conclude with a 
summary of our results in section IV where we use our expressions to calculate the polarization as a function of the distance from the center of an isotropic atomic trap, for typical trap parameters. We also identify the region where inhomogeneous phases may be found in a typical trap geometry.

\section{THE MODEL LAGRANGIAN}

We describe the gas of two species of fermions, $\psi_{1}$ and $\psi_{2}$, with chemical potentials $\mu_{1}$ and $\mu_{2}$, at finite temperature $T$, by a model lagrangian density of the form,

$$
\mathcal{L}=\psi_{\alpha}^{\dagger}\left(\left(i \partial_{t}-\xi(\hat{\mathbf{p}})\right) \delta_{\alpha \beta}+\delta \mu \sigma_{\alpha \beta}^{3}\right) \psi_{\beta}+\frac{\lambda}{2} \psi_{\alpha}^{\dagger} \psi_{\beta}^{\dagger} \psi_{\beta} \psi_{\alpha},
$$

where $\xi(\hat{\mathbf{p}})=\hat{\mathbf{p}}^{2} /(2 m)-\mu$. The chemical potentials for the two species of fermions, $\psi_{1}$ and $\psi_{2}$, in terms of the average chemical potential $\mu$ and the splitting $2 \delta \mu$ are, $\mu_{1}=\mu+\delta \mu$ and $\mu_{2}=\mu-\delta \mu$, respectively. We are interested in the case where the interaction between the two species of fermions is attractive, meaning $\lambda>0$. In the BCS regime, then, for small enough $T$ and $\delta \mu$, the phase of the system will be characterized by a non-zero difermion condensate

$$
\left\langle\psi_{\alpha}(\mathbf{r}) \psi_{\beta}(\mathbf{r})\right\rangle=\frac{1}{\lambda} \epsilon_{\alpha \beta} \Delta(\mathbf{r}) .
$$

We are interested in the phase boundary between such a superfluid phase and the normal phase, where $\Delta(\mathbf{r})=0$.

In addition, the system will be characterized by specific profiles for the number density of the two species ,

$$
\left\langle\psi_{1}^{\dagger}(\mathbf{r}) \psi_{1}(\mathbf{r})\right\rangle=n_{1}(\mathbf{r}), \quad\left\langle\psi_{2}^{\dagger}(\mathbf{r}) \psi_{2}(\mathbf{r})\right\rangle=n_{2}(\mathbf{r}) .
$$

In our calculations we will find it more convenient to write $n_{1}$ and $n_{2}$ in terms of the average density $n(r)=(1 / 2)\left(n_{1}(\mathbf{r})+n_{2}(\mathbf{r})\right)$ and the difference in densities $\delta n(r)=(1 / 2)\left(n_{1}(\mathbf{r})-n_{2}(\mathbf{r})\right)$.

To specify the system for any $T, \mu, \delta \mu$ for a given $\lambda$ (which we will trade for the scattering length $a$ ), we need three equations that let us solve for the three variables $\Delta, n$ and $\delta n$. These are the gap and the number equations. For homogeneous condensates these take the form,

$$
\frac{\partial \Omega}{\partial \Delta}=0, \quad \frac{\partial \Omega}{\partial \mu}=-2 n, \quad \frac{\partial \Omega}{\partial \delta \mu}=-2 \delta n .
$$

We calculate the free energy, $\Omega$, in a mean field approximation where we replace the four Fermi interaction by its mean field value

$$
\begin{aligned}
\frac{\lambda}{2} \psi_{\alpha}^{\dagger}(\mathbf{r}) \psi_{\beta}^{\dagger}(\mathbf{r}) \psi_{\beta}(\mathbf{r}) \psi_{\alpha}(\mathbf{r}) \rightarrow & \frac{1}{2} \Delta^{*}(\mathbf{r}) \epsilon_{\alpha \beta} \psi_{\alpha}(\mathbf{r}) \psi_{\beta}(\mathbf{r})-\frac{1}{2} \Delta(\mathbf{r}) \epsilon_{\alpha \beta} \psi_{\alpha}^{\dagger}(\mathbf{r}) \psi_{\beta}^{\dagger}(\mathbf{r})-\frac{|\Delta(\mathbf{r})|^{2}}{\lambda} \\
& +\lambda n_{1}(\mathbf{r}) \psi_{2}^{\dagger}(\mathbf{r}) \psi_{2}(\mathbf{r})+\lambda n_{2}(\mathbf{r}) \psi_{1}^{\dagger}(\mathbf{r}) \psi_{1}(\mathbf{r})-\lambda n_{1}(\mathbf{r}) n_{2}(\mathbf{r}) .
\end{aligned}
$$

The terms proportional to $n_{1}$ and $n_{2}$ give rise to Hartree corrections to the free energy.

Upon making the mean field approximation, and performing standard manipulations, we can write the lagrangian density (eq. (3)) in a quadratic form in terms of the Nambu-Gorkov spinor,

$$
\Psi=\left(\begin{array}{llll}
\psi_{1} & \psi_{2} & \psi_{1}^{\dagger} & \psi_{2}^{\dagger}
\end{array}\right)^{T} .
$$

The final answer is

$$
\mathcal{L}=\frac{1}{2} \Psi^{\dagger}\left(\begin{array}{cc}
i \partial_{t}-\tilde{\xi}(\mathbf{p})+\delta \tilde{\mu} \sigma^{3} & -\Delta(x) \varepsilon \\
\Delta^{*}(x) \varepsilon & i \partial_{t}+\tilde{\xi}(\mathbf{p})-\delta \tilde{\mu} \sigma^{3}
\end{array}\right) \Psi+\delta(\mathbf{0}) \tilde{\mu}-\frac{|\Delta|^{2}}{\lambda}-\lambda\left(n^{2}(\mathbf{r})-\delta n^{2}(\mathbf{r})\right),
$$


where $\tilde{\xi}(\mathbf{p})=\hat{\mathbf{p}}^{2} /(2 m)-\tilde{\mu}$. $\tilde{\mu}$ and $\delta \tilde{\mu}$ include the Hartree terms and are given by

$$
\tilde{\mu}=\mu+\lambda n, \quad \delta \tilde{\mu}=\delta \mu-\lambda \delta n .
$$

That the Hartree corrections to the chemical potentials should be this way can be seen immediately if we recall that the Hartree terms change $\mu_{1}$ to $\tilde{\mu}_{1}=\mu_{1}+\lambda n_{2}$ and $\mu_{2}$ to $\tilde{\mu}_{2}=\mu_{2}+\lambda n_{1}$. The presence of the mysterious looking term $\delta(\mathbf{0}) \tilde{\mu}$ can be explained as follows.

When written in terms of the fields $\psi$ and $\psi^{\dagger}$, the mean field lagrangian density has a piece $\sum_{\alpha} \tilde{\mu}_{\alpha} \psi_{\alpha}^{\dagger} \psi_{\alpha}$. To write this in a symmetric form in terms of the components of the Nambu-Gorkov spinor $\Psi$, we need to exchange the ordering of $\psi$ and $\psi^{\dagger}$, which gives rise to the term in question. More explicitly,

$$
\sum_{\alpha} \tilde{\mu}_{\alpha} \psi_{\alpha}^{\dagger} \psi_{\alpha}=\frac{1}{2} \sum_{\alpha}\left(\tilde{\mu}_{\alpha} \psi_{\alpha}^{\dagger} \psi_{\alpha}+\tilde{\mu}_{\alpha} \psi_{\alpha}^{\dagger} \psi_{\alpha}\right)=\frac{1}{2} \sum_{\alpha}\left(\tilde{\mu}_{\alpha} \psi_{\alpha}^{\dagger} \psi_{\alpha}-\tilde{\mu}_{\alpha} \psi_{\alpha} \psi_{\alpha}^{\dagger}+\tilde{\mu}_{\alpha} \delta(\mathbf{0})\right),
$$

where we have used the fermion anticommutation relation

$$
\left\{\psi_{\alpha}(\mathbf{r}), \psi_{\beta}^{\dagger}\left(\mathbf{r}^{\prime}\right)\right\}=\delta_{\alpha \beta} \delta\left(\mathbf{r}-\mathbf{r}^{\prime}\right) .
$$

This term is important in canceling out a divergent contribution to the free energy, as we shall see below. A similar term occurs while reordering $\sum_{\alpha} \psi_{\alpha}^{\dagger}\left(i \partial_{t}-\hat{\mathbf{p}}^{2} /(2 m)\right) \psi_{\alpha}$, but is the same in normal and superfluid matter and does not affect the phase competition.

In the mean field approximation the lagrangian density is bilinear in the fermion fields and the free energy is found by direct integration over the fields. We find

$$
\begin{aligned}
\int d^{4} x_{E} \Omega= & \int d^{4} x_{E}\left\{-\delta(\mathbf{0}) \tilde{\mu}+\frac{|\Delta(\mathbf{r})|^{2}}{\lambda}+\lambda\left(n^{2}(\mathbf{r})-\delta n^{2}(\mathbf{r})\right)\right\} \\
& -\left\{\left[\frac{1}{2} \operatorname{Tr} \log \left(\begin{array}{cc}
-\partial_{x^{4}}-\tilde{\xi}(\mathbf{p})+\delta \tilde{\mu} & -\Delta(\mathbf{r}) \\
-\Delta^{*}(\mathbf{r}) & -\partial_{x^{4}}+\tilde{\xi}(\mathbf{p})+\delta \tilde{\mu}
\end{array}\right)+(\delta \tilde{\mu} \rightarrow-\delta \tilde{\mu})\right]\right\}
\end{aligned}
$$

where $x_{E}$ represents the euclidean space four vector $\left(x^{4}, \mathbf{r}\right)$, with $\mathbf{r}$ lying in position space with volume $V$ and $x^{4} \in[-1 /(2 T), 1 /(2 T)]$.

We now compare the free energies of the superfluid phases to the normal phase to find where the boundary between the phases lies.

\section{PHASE BOUNDARY BETWEEN THE SUPERFLUID AND NORMAL PHASE}

We first consider the competition between the homogeneous superfluid phase and the normal phase, and then the competition between inhomogeneous superfluids and the normal phase.

\section{A. Homogeneous superfluid and the normal phase}

The homogeneous phases are defined by the condition that $\Delta, n$ and $\delta n$ are all independent of $\mathbf{r}$. The argument of the log in eq. (13) is then diagonal in momentum space and the free energy density is simply,

$$
\begin{aligned}
\Omega(\Delta, n, \delta n, T, \mu, \delta \mu)= & \left.-\tilde{\mu} \int \tilde{d}^{3} \mathbf{p}(1)+\frac{|\Delta|^{2}}{\lambda}+\lambda\left(n^{2}-\delta n^{2}\right)\right\} \\
& -\left\{\int \tilde{d}^{3} \mathbf{p}\left[\frac{T}{2} \sum_{\substack{p^{4}=\\
(2 n+1) \pi T}} \log \left(\left(i p^{4}+\delta \tilde{\mu}-\tilde{\epsilon}(\mathbf{p})\right)\left(i p^{4}+\delta \tilde{\mu}+\tilde{\epsilon}(\mathbf{p})\right)\right)+(\delta \tilde{\mu} \rightarrow-\delta \tilde{\mu})\right]\right\} \\
= & \left\{\frac{|\Delta|^{2}}{\lambda}+\lambda\left(n^{2}(\mathbf{r})-\delta n^{2}(\mathbf{r})\right)\right\} \\
& -\int \tilde{d}^{3} \mathbf{p}\left\{T\left[\log \left[\cosh \left(\frac{\delta \tilde{\mu}+\tilde{\epsilon}(\mathbf{p})}{2 T}\right)\right]+\log \left[\cosh \left(\frac{-\delta \tilde{\mu}+\tilde{\epsilon}(\mathbf{p})}{2 T}\right)\right]\right]+\tilde{\mu}\right\},
\end{aligned}
$$


where $\tilde{\epsilon}(\mathbf{p})=\sqrt{ }\left(\tilde{\xi}^{2}(\mathbf{p})+|\Delta|^{2}\right)$ and we have rewritten $\delta(\mathbf{0})$ as an intergal over momentum space.

The gap equation is the condition that the free energy is stationary with respect to small variations in the magnitude of $\Delta$. (A position independent phase of $\Delta$ does not affect the free energy and hence, for simplicity, we will take $\Delta$ to be real and positive for the rest of the section (IIIA).)

$$
\begin{aligned}
0 & =\frac{\partial \Omega}{\partial \Delta} \\
& =\frac{2 \Delta}{\lambda}-\frac{1}{2} \int \tilde{d}^{3} \mathbf{p} \frac{\Delta}{\tilde{\epsilon}(\mathbf{p})}\left[\tanh \left(\frac{\delta \tilde{\mu}+\tilde{\epsilon}(\mathbf{p})}{2 T}\right)+\tanh \left(\frac{-\delta \tilde{\mu}+\tilde{\epsilon}(\mathbf{p})}{2 T}\right)\right] .
\end{aligned}
$$

The trivial solution, $\Delta=0$, corresponds to the normal phase. A competing superfluid phase exists if eq. (15) has a solution with $\Delta \neq 0$.

As it stands, the second term in eq. (15) is linearly divergent in $\mathbf{p}$ and needs to be regulated in some manner. One way is to cut off the momentum integration at some momentum $\Lambda$, chosen to be sufficiently larger than the Fermi momentum, $k_{F}=\sqrt{ }(2 m \mu)$, to capture all the features of the intergand. In the weak coupling (BCS) regime, it enough to take $\Lambda$ to be several times $k_{F}$. The solution of eq. (15) then depends on $\lambda$ and $\Lambda$, but it is useful to rewrite the results in terms of a physical observable. A popular choice is to use the relation between the $s$-wave scattering length $a$, and $\lambda$.

$$
\frac{1}{\lambda}=\frac{-m}{4 \pi a}+\int d^{3} \mathbf{p} \frac{m}{\mathbf{p}^{2}}
$$

Canceling $2 \Delta \neq 0$ from the right hand side of the gap equation, eq. (15), and substituting $1 / \lambda$ in the first term in eq. (15) from eq. (16), we obtain the relation

$$
-\frac{m}{4 \pi a}=\int \tilde{d}^{3} \mathbf{p}\left\{\frac{1}{4 \tilde{\epsilon}(\mathbf{p})}\left[\tanh \left(\frac{\delta \tilde{\mu}+\tilde{\epsilon}(\mathbf{p})}{2 T}\right)+\tanh \left(\frac{-\delta \tilde{\mu}+\tilde{\epsilon}(\mathbf{p})}{2 T}\right)\right]-\frac{m}{\mathbf{p}^{2}}\right\},
$$

which is now cutoff independent. Recall, however, that $\lambda$ also appears implicitly in the definitions of $\tilde{\mu}$ and $\delta \tilde{\mu}$ (eq. (10)) where it multiplies $n$ and $\delta n$, respectively. In all these places, we replace $\lambda$ by $-(4 \pi a) / m$. To see why this is reasonable, consider eq. (16) with a momentum cut off $\Lambda$.

$$
\frac{1}{\lambda}=-\frac{m}{4 \pi a}+m \Lambda \text {. }
$$

If $|4 \pi \Lambda a| \ll 1$, then in eq. (18) we can ignore the second term on the right hand side compared to the first term. In the weak coupling limit $\left|\pi k_{F} a\right| \ll 1$. If $\Lambda$ is taken to be not many times larger than $k_{F}$, as we argued can be done in the weak coupling regime, then indeed we can take $\lambda$ to be $-(4 \pi a) / m$ to a good approximation.

To summarize, the gap equation is given by eq. (17) with

$$
\tilde{\mu}=\mu+\left(\frac{-4 \pi a}{m}\right) n, \quad \delta \tilde{\mu}=\delta \mu-\left(\frac{-4 \pi a}{m}\right) \delta n .
$$

The number equations are found by explicitly calculating the derivatives with respect to $\mu$ and $\delta \mu$ (eq. (6)). The final expressions are given below.

$$
-n=\frac{1}{4} \int \ddot{d}^{3} \mathbf{p}\left\{\left[\tanh \left(\frac{\delta \tilde{\mu}+\tilde{\epsilon}(\mathbf{p})}{2 T}\right)+\tanh \left(\frac{-\delta \tilde{\mu}+\tilde{\epsilon}(\mathbf{p})}{2 T}\right)\right] \frac{\tilde{\xi}(\mathbf{p})}{\tilde{\epsilon}(\mathbf{p})}-2\right\},
$$

and,

$$
-\delta n=\frac{1}{4} \int \tilde{d}^{3} \mathbf{p}\left\{\left[-\tanh \left(\frac{\delta \tilde{\mu}+\tilde{\epsilon}(\mathbf{p})}{2 T}\right)+\tanh \left(\frac{-\delta \tilde{\mu}+\tilde{\epsilon}(\mathbf{p})}{2 T}\right)\right]\right\},
$$


with $\tilde{\mu}$ and $\delta \tilde{\mu}$ given by eq. (19).

For the normal phase, $\Delta=0$, and we can solve eqs. (20121) to find $n$ and $\delta n$ to obtain values we will call $n_{N}$ and $\delta n_{N}$ respectively. The free energy of the normal phase is given by,

$$
\begin{aligned}
\Omega_{N}(\mu, \delta \mu, T)= & \left\{\left(\frac{-4 \pi a}{m}\right)\left(n_{N}^{2}-\delta n_{N}^{2}\right)\right\} \\
& -\int \vec{d}^{3} \mathbf{p}\left\{T\left[\log \left[\cosh \left(\frac{\delta \tilde{\mu}_{N}+\tilde{\xi}(\mathbf{p})_{N}}{2 T}\right)\right]+\log \left[\cosh \left(\frac{-\delta \tilde{\mu}_{N}+\tilde{\xi}(\mathbf{p})_{N}}{2 T}\right)\right]\right]+\tilde{\mu}_{N}\right\}
\end{aligned}
$$

with $\tilde{\xi}(\mathbf{p})_{N}=\hat{\mathbf{p}}^{2} /(2 m)-\tilde{\mu}_{N}$ and

$$
\tilde{\mu}_{N}=\mu+\left(\frac{-4 \pi a}{m}\right) n_{N}, \quad \delta \tilde{\mu}_{N}=\delta \mu-\left(\frac{-4 \pi a}{m}\right) \delta n_{N} .
$$

If, in addition, eqs. (17/20[21) possess solutions with $\Delta \neq 0, n_{\Delta}$ and $\delta n_{\Delta}$, we need to compare the free energies of these superfluid solutions to $\Omega_{N}$. The difference in the free energies is given by

$$
\begin{aligned}
\left(\Omega_{s}-\Omega_{N}\right)(\mu, \delta \mu, T)=\{ & \left.-\frac{m \Delta^{2}}{4 \pi a}+\left(\frac{-4 \pi a}{m}\right)\left(n_{\Delta}^{2}-n_{N}^{2}-\delta n_{\Delta}^{2}+\delta n_{N}^{2}\right)\right\} \\
& -\int \vec{d}^{3} \mathbf{p}\left\{T\left[\log \left[\cosh \left(\frac{\delta \tilde{\mu}+\tilde{\epsilon}(\mathbf{p})}{2 T}\right)\right]+\log \left[\cosh \left(\frac{-\delta \tilde{\mu}+\tilde{\epsilon}(\mathbf{p})}{2 T}\right)\right]\right]+\tilde{\mu}-\frac{m \Delta^{2}}{\mathbf{p}^{2}}\right\} .
\end{aligned}
$$

If $\Omega_{s}-\Omega_{N}>0$ then the normal phase is favored over the superfluid phase, and vice versa. In Fig. (1) we look at the boundary marking the normal to superfluid phase transition in $T, \delta \mu$ space for $g=\left(\pi k_{F} a\right)^{(-1)}=$ -0.72 . For different values of $\mu$ and $m$, if we choose $a$ so that the dimensionless parameter $g$ remains the same, then the physical quantities scale with $\mu$ and $m$ as follows.

$$
\begin{aligned}
n_{\alpha}(m, \mu, T, \delta \mu) & =m^{(3 / 2)} \mu^{(3 / 2)} n_{\alpha}(1,1,(T / \mu),(\delta \mu / \mu)) \\
\Delta(m, \mu, T, \delta \mu) & =\mu \Delta(1,1,(T / \mu),(\delta \mu / \mu)) \\
\Omega(m, \mu, T, \delta \mu) & =m^{(3 / 2)} \mu^{(5 / 2)} \Omega(1,1,(T / \mu),(\delta \mu / \mu)) .
\end{aligned}
$$

In particular, the value of $\Delta_{0}$, the value of the gap parameter at $T=0$ and $\delta \mu=0$, scales linearly with $\mu$. To remove the dependence of the phase transition curve on the overall scales, it is useful to draw the phase diagram in terms of dimensionless variables. Since $\Delta_{0}$ is proportional to $\mu$, we take the $x$-axis to be $\delta \mu / \Delta_{0}$ and the $y$-axis to be $T / \Delta_{0}$.

For comparison, in Fig. (11) we also show the result when one does not include the Hartree corrections. One effect of Hartree corrections is to simply shift the chemical potentials. For example, at $T=0$ and $\delta \mu=0$, including these increases the "effective" chemical potential, $\tilde{\mu}=\mu-(4 \pi a / m) n$. (Recall that $a<0$ in the BCS regime.) Therefore, for the same $a$, the value of $\Delta_{0}$ is greater when we include the Hartree corrections, compared to when we do not include them. To get rid of this overall change in $\Delta_{0}$, we scale $T$ and $\delta \mu$ in the "non-Hartree" curve by the "non-Hartree" value of $\Delta_{0}$. The two curves are still different, and that has to do with the fact that including Hartree corrections affects the competition between the normal and the superfluid phases. Number densities, and therefore Hartree corrections, are different in the two phases at the phase boundary, if the phase transition between the two phases is first order. In the following paragraphs, we discuss the effect of Hartree corrections on the phase transition curve between the normal and the superfluid phase in more detail.

First looking at $T=0, \delta \mu / \Delta_{0}$ at the first order transition is larger than the weak coupling value 0.707 , as pointed out earlier in [7]. Since at $T=0, \delta n=0$ in the superfluid phase (the superfluid phase is gapped), this is not simply due to a reduction in the effective splitting between the Fermi surfaces in the superfluid region $\left(\delta \tilde{\mu}=\delta \mu \sim 0.81 \Delta_{0}\right.$ for the superfluid phase at the phase transition). As discussed above, the change is due to the fact that $n$ and $\delta n$ change abruptly at the first order phase boundary. More specifically, there 
are two effects both of which drive the phase transition to larger $\delta \mu$. Firstly, $\delta n$ is positive in the normal phase, implying $\delta \tilde{\mu}$ is smaller than $\delta \mu$, which increases the free energy of the normal phase. Secondly, $n$ is larger in the superfluid phase because of pairing, and this also makes the superfluid phase more favorable.

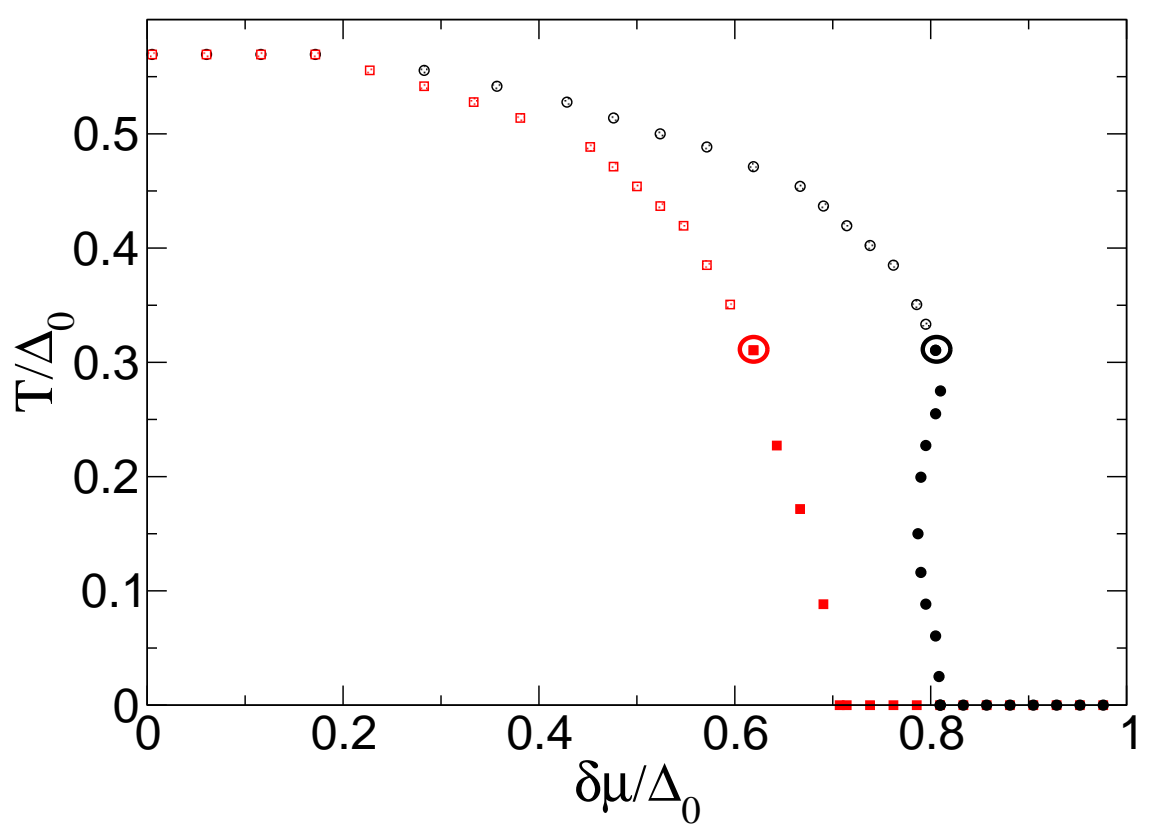

FIG. 1: (colored online) We show the phase boundary between normal and homogeneous superfluid phases in $\delta \mu, T$ space, for $g=-0.72$. In the curve marked by circles (black online), we include the Hartree corrections. The value of $\Delta_{0} / \mu$ is 0.058 . The curve marked by squares (red online) does not include Hartree corrections. The value of $\Delta_{0} / \mu$ in this case is 0.03. At $T=0$, the first order phase transition from the superfluid to the normal phase occurs at $\delta \mu / \Delta_{0} \sim 0.71$, consistent with weak coupling results. The filled points mark the region where the phase transition is first order while the hollow points mark a second order transition. The points where the transition changes from being second order to becoming first order have been encircled.

Next, looking along the $y$-axis, at $\delta \mu=0$, the transition from the superfluid to the normal phase at the critical temperature is second order, and the number densities in the two phases are the same at the critical temperature. It is understandable, therefore, that the transition occurs at $T_{c} / \Delta_{0} \sim 0.567$, the standard weak coupling value.

Things are interesting close to $\delta \mu / \Delta_{0} \sim 0.8$, where the shape of the curve is qualitatively altered. For a window of splittings, $\delta \mu / \Delta_{0} \in(0.79,0.81)$, as we increase the temperature, we encounter not one but three normal-superfluid transitions. To clarify how this comes about, consider the shape of $\Omega$ as a function of $\Delta$ for various values of the temperature, at $\delta \mu / \Delta_{0}=0.795$ (Fig. 21). At $T=0$, the local minimum at $\Delta=\Delta_{0}$ is favored. As we increase the temperature, this minimum becomes shallower and eventually there is a first order transition to the normal phase. As we keep increasing the temperature, the $\Delta=0$ solution becomes unstable and the superfluid state is favored again for a range of temperatures. Eventually, at a large enough temperature, there is a second order transition to the normal phase. This reappearance of superfluidity at higher temperatures can be understood intuitively as follows. At zero temperature, BCS pairing is stressed 


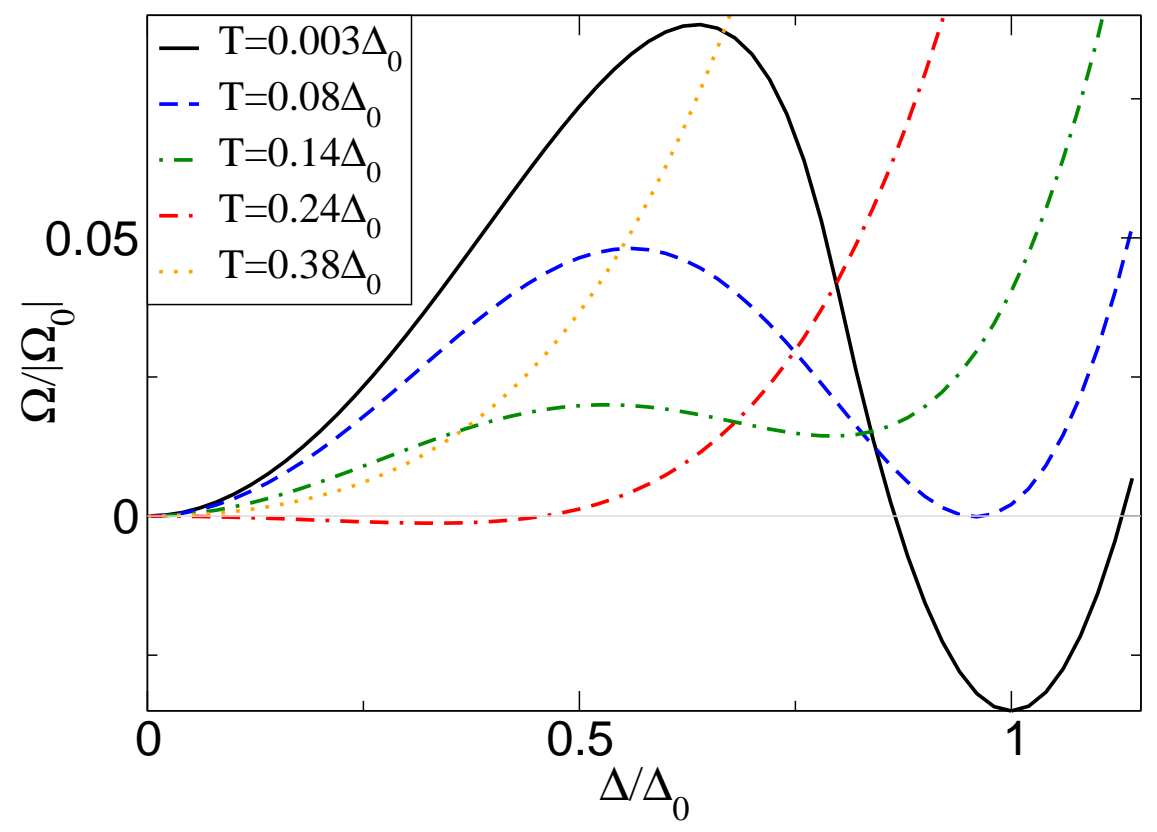

FIG. 2: (colored online) This figure shows the free energy as a function of $\Delta$ at $\delta \mu / \Delta_{0}=0.795$ for various values of $T$. The solid curve (black online) corresponds to the lowest temperature, $T / \Delta_{0}=0.003$, and clearly shows a robust superfluid phase with $\Delta \sim \Delta_{0}$. The dashed curve (blue online) corresponds to $T / \Delta_{0}=0.08$ and is the point of a first order phase transition to the normal phase. A "fragile" superfluid phase reappears before it disappears again at a higher temperature.

due to a non-zero $\delta \mu$, because fermions of the two species can not find partners of opposite momenta lying on the distinct Fermi surfaces determined by their different chemical potentials. BCS pairing in such systems requires the two distinct Fermi spheres to equalize at a Fermi momentum different from the value given by the corresponding chemical potentials, costing free energy. At non-zero temperatures, however, the Fermi surfaces are smeared and it is possible to find partners of opposite momenta even without equalizing the Fermi surfaces. Such an effect of temperature on pairing has been noted previously in the context of pairing between $u$ and $d$ quarks in the 2SC phase [22, 23, 24, 25, 26, 27, 28], and a nuclear physics context [29].

To see in a different way why there are three transitions, it is useful to expand the free energy in powers of $\Delta^{2}$ (Ginzburg-Landau expansion) and look at how the coefficient of the quadratic term changes as we change $T$, keeping $\delta \mu$ constant. We write the free energy as $\alpha \Delta^{2}+\mathcal{O}\left(\Delta^{4}\right) . \alpha<0$ points to an instability in the $\Delta=0$ state to the formation of a non-zero condensate, while $\alpha>0$ means that the normal state is locally stable, but does not tell us whether it is globally favored or not.

In Fig. (3) we plot $\alpha$ (in units of $\Delta_{0}^{2}$ ) as a function of $T / \Delta_{0}$, for $\delta \mu=0.795 \Delta_{0}$ kept constant. For small $T$, the normal phase is locally stable, but globally disfavored to the $\Delta=\Delta_{0}$ state (Fig. (2)). As we increase $T$, the superfluid phase becomes less and less favorable until finally at $T / \Delta_{0}=0.08$ the normal phase is globally favored. On increasing $T$ further, we find that the normal phase becomes locally unstable in the region $T / \Delta_{0} \in(0.22,0.33)$, and this gives rise to a region of "fragile" superfluidity.

For smaller couplings the window of $\delta \mu / \Delta_{0}$ where we encounter three normal-superfluid transitions, becomes narrower, while for stronger couplings it becomes wider. 


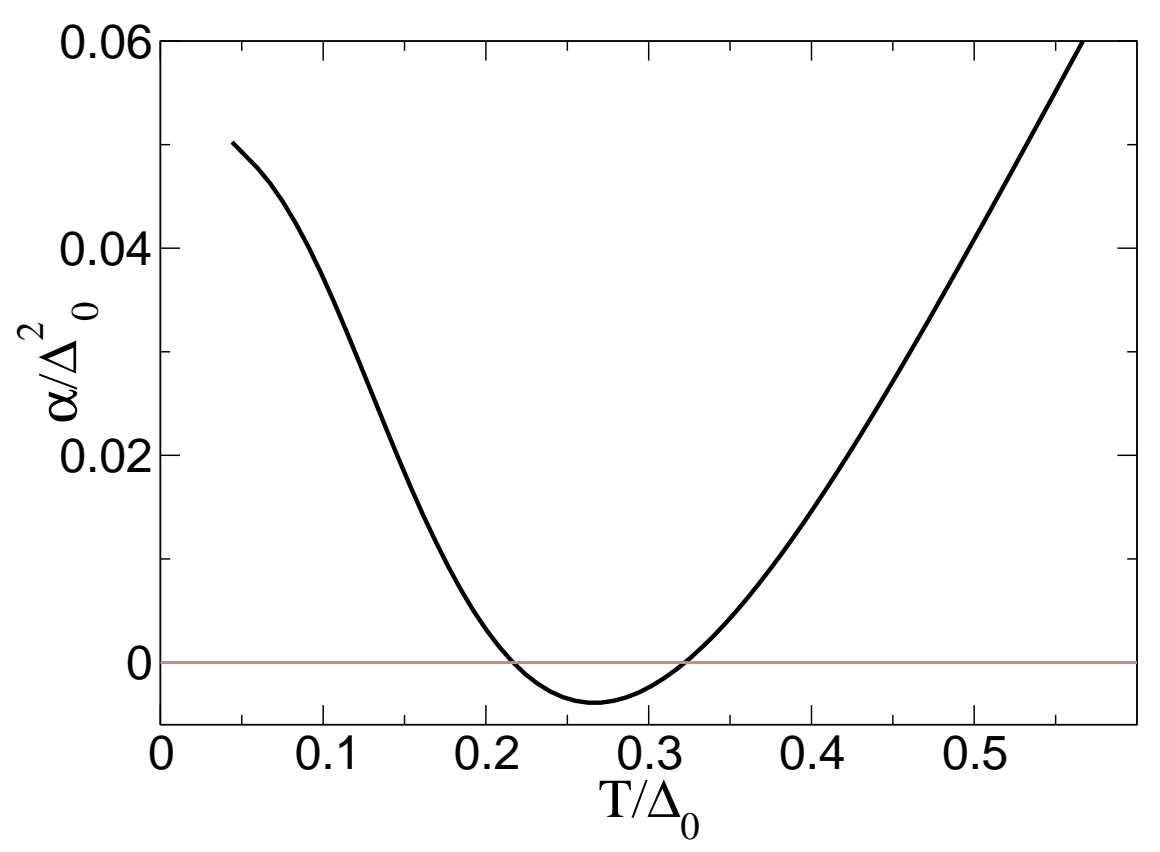

FIG. 3: Plot of $\alpha$ as a function of $T / \Delta_{0}$ for $\delta \mu=0.795 \Delta_{0}$. The normal phase is locally stable for $T / \Delta_{0} \in(0,0.22)$, locally unstable for $T / \Delta_{0} \in(0.22,0.33)$, and locally stable for $T$ larger than $0.33 \Delta_{0}$.

\section{B. Inhomogeneous condensates}

Let us now consider the case where $\Delta, n$ and $\delta n$ depend on $\mathbf{r}$. The argument of the log in eq. (13) is no longer diagonal in momentum space and hence we evaluate the free energy in a Ginzburg-Landau expansion. We are interested in finding the curve along which the normal phase becomes unstable to the growth of an inhomogeneous condensate. Working in the limit of small $\Delta(\mathbf{r})$, we expand the log in $\Delta(\mathbf{r})$ and drop terms proportional to $\Delta^{4}$ and higher, and obtain

$$
\begin{aligned}
\int d^{3} \mathbf{r} \Omega=\int d^{3} \mathbf{r}\left\{-\tilde{\mu} \int d^{3} \mathbf{p}(1)+\frac{|\Delta(\mathbf{r})|^{2}}{\lambda}+\lambda\left(n^{2}-\delta n^{2}\right)\right\} \\
-\left\{T \sum _ { \substack { p ^ { 4 } = \\
( 2 n + 1 ) \pi T } } \left[\operatorname{Tr}_{\mathbf{r}} \log \left(\left(i p^{4}+\delta \tilde{\mu}-\tilde{\xi}(\mathbf{p})\right)\left(i p^{4}+\delta \tilde{\mu}+\tilde{\xi}(\mathbf{p})\right)\right)\right.\right. \\
\left.\left.\quad-\operatorname{Tr}_{\mathbf{r}}\left(\left(i p^{4}+\delta \tilde{\mu}-\tilde{\xi}(\mathbf{p})\right)^{(-1)} \Delta(\mathbf{r})\left(i p^{4}+\delta \tilde{\mu}+\tilde{\xi}(\mathbf{p})\right)^{(-1)} \Delta^{*}(\mathbf{r})\right)\right]\right\}
\end{aligned}
$$

We now argue that within the approximations we are working in, we can replace $n$ and $\delta n$ by their values in normal matter to compute $\Omega$. It is obvious that we can do so in the term,

$$
T \sum_{\substack{p^{4}=\\(2 n+1) \pi T}}\left(\left(i p^{4}+\delta \tilde{\mu}-\tilde{\xi}(\mathbf{p})\right)^{(-1)} \Delta(\mathbf{r})\left(i p^{4}+\delta \tilde{\mu}+\tilde{\xi}(\mathbf{p})\right)^{(-1)} \Delta^{*}(\mathbf{r})\right)
$$

because the corrections to $n$ and $\delta n$ due to pairing is proportional to $\Delta^{2}$, and keeping these corrections in eq. (27) (where they appear in $\tilde{\mu}$ and $\delta \tilde{\mu}$ ) will only change the result by order $\Delta^{4}$. There are $\mathcal{O}\left(\Delta^{2}\right)$ contributions to $\Omega$ from $\tilde{\mu} \int \tilde{d}^{3} \mathbf{p}(1), \lambda\left(n^{2}-\delta n^{2}\right)$ and $\operatorname{Tr}_{\mathbf{r}} \log \left(\left(i p^{4}+\delta \tilde{\mu}-\tilde{\xi}(\mathbf{p})\right)\left(i p^{4}+\delta \tilde{\mu}+\tilde{\xi}(\mathbf{p})\right)\right)$, but in all these cases, the $\Delta^{2}$ correction to $n$ or $\delta n$ is further multiplied by $\lambda$ and for weak coupling, this should give 
a small overall contribution. (This is the $\Delta^{2}$ correction to the Hartree term which itself is a correction). In the approximation where we neglect this contribution, when we calculate the difference between the normal and superfluid free energies, these three terms cancel out and we obtain

$$
\begin{aligned}
\int d^{3} \mathbf{r}\left(\Omega(\Delta(\mathbf{r}))-\Omega_{N}\right)= & d d^{3} \mathbf{r}\left\{\frac{|\Delta(\mathbf{r})|^{2}}{\lambda}\right\} \\
& +\int d^{3} \mathbf{p}\left\{T \sum_{\substack{p^{4}=\\
(2 n+1) \pi T}}\left[\operatorname{Tr}_{\mathbf{r}}\left(\left(i p^{4}+\delta \tilde{\mu}_{N}-\tilde{\xi}(\mathbf{p})_{N}\right)^{(-1)} \Delta(\mathbf{r})\left(i p^{4}+\delta \tilde{\mu}_{N}+\tilde{\xi}(\mathbf{p})_{N}\right)^{(-1)} \Delta^{*}(\mathbf{r})\right)\right]\right\}
\end{aligned}
$$

In momentum space this gives,

$$
\begin{aligned}
\int d^{3} \mathbf{r}\left(\Omega(\Delta(\mathbf{r}))-\Omega_{N}\right)= & \int \tilde{d}^{3} \mathbf{k} \Delta(\mathbf{k}) \Delta^{*}(-\mathbf{k})\left[\frac{-m}{4 \pi a}+\right. \\
& \left.+\int \tilde{d}^{3} \mathbf{p}\left\{\frac{m}{\mathbf{p}^{2}}+T \sum_{\substack{p^{4}=\\
(2 n+1) \pi T}}\left(i p^{4}+\delta \tilde{\mu}_{N}-\tilde{\xi}(\mathbf{p}+\mathbf{k})_{N}\right)^{(-1)}\left(i p^{4}+\delta \tilde{\mu}_{N}+\tilde{\xi}(\mathbf{p})_{N}\right)^{(-1)}\right\}\right],
\end{aligned}
$$

where we have used eq. (16) to rewrite $\lambda$.

It is convenient at this point, to separate the "potential" contribution (the contribution independent of $\mathbf{k}$ ) from the "gradient" contribution (zero for $\mathbf{k}=\mathbf{0}$ ) [30]. We write

$$
\int d^{3} \mathbf{r}\left(\Omega(\Delta(\mathbf{r}))-\Omega_{N}\right)=\int \mathbb{d}^{3} \mathbf{k} \Delta(\mathbf{k}) \Delta^{*}(-\mathbf{k})\{\alpha+f(|\mathbf{k}|)\}
$$

with,

$$
\begin{aligned}
\alpha & =-\frac{m}{4 \pi a}+\int \tilde{d}^{3} \mathbf{p}\left\{\frac{m}{\mathbf{p}^{2}}+T \sum_{\substack{p^{4}=\\
(2 n+1) \pi T}}\left(i p^{4}+\delta \tilde{\mu}_{N}-\tilde{\xi}(\mathbf{p})_{N}\right)^{(-1)}\left(i p^{4}+\delta \tilde{\mu}_{N}+\tilde{\xi}(\mathbf{p})_{N}\right)^{(-1)}\right\} \\
& =-\frac{m}{4 \pi a}+\int \tilde{d}^{3} \mathbf{p}\left\{\frac{m}{\mathbf{p}^{2}}-\left[\tanh \left(\frac{\delta \tilde{\mu}_{N}+\tilde{\xi}(\mathbf{p})_{N}}{2 T}\right)+\tanh \left(\frac{-\delta \tilde{\mu}_{N}+\tilde{\xi}(\mathbf{p})_{N}}{2 T}\right)\right] \frac{1}{4 \xi(\mathbf{p})}\right\}
\end{aligned}
$$

and,

$$
\begin{aligned}
f(|\mathbf{k}|) & =\frac{1}{2} \int \pi^{3} \mathbf{p}\left\{T \sum_{\substack{p^{4}=\\
(2 n+1) \pi T}} \frac{\left(\tilde{\xi}(\mathbf{p}+\mathbf{k})_{N}-\tilde{\xi}(\mathbf{p})_{N}\right)^{2}}{\left(\left(i p^{4}+\delta \tilde{\mu}_{N}\right)^{2}-\left(\tilde{\xi}(\mathbf{p})_{N}\right)^{2}\right)\left(\left(i p^{4}+\delta \tilde{\mu}_{N}\right)^{2}-\left(\tilde{\xi}(\mathbf{p}+\mathbf{k})_{N}\right)^{2}\right)}\right\} \\
& =\frac{1}{2} \int \pi^{3} \mathbf{p}\left\{\frac{\left(\tilde{\xi}(\mathbf{p}+\mathbf{k})_{N}-\tilde{\xi}(\mathbf{p})_{N}\right)}{\left(\tilde{\xi}(\mathbf{p}+\mathbf{k})_{N}+\tilde{\xi}(\mathbf{p})_{N}\right)}\left[\frac{g\left(\tilde{\xi}(\mathbf{p})_{N}\right)}{\tilde{\xi}(\mathbf{p})_{N}}-\frac{g\left(\tilde{\xi}(\mathbf{p}+\mathbf{k})_{N}\right)}{\tilde{\xi}(\mathbf{p}+\mathbf{k})_{N}}\right]\right\},
\end{aligned}
$$

where

$$
g(\xi)=\frac{1}{2}\left[\tanh \left(\frac{\delta \tilde{\mu}+\xi}{2 T}\right)+\tanh \left(\frac{-\delta \tilde{\mu}+\xi}{2 T}\right)\right] .
$$

At any given temperature, for large enough $\delta \mu$, the normal phase will be favored over a phase with nonzero $\Delta(\mathbf{k})$, and the combination $\alpha+f(k)$ will be positive for all values of $k=|\mathbf{k}|$. As we decrease $\delta \mu$ keeping $T$ constant, $\alpha+f(k)$ may become zero, and then negative, for a single mode with momentum $k=k_{\min }$. 


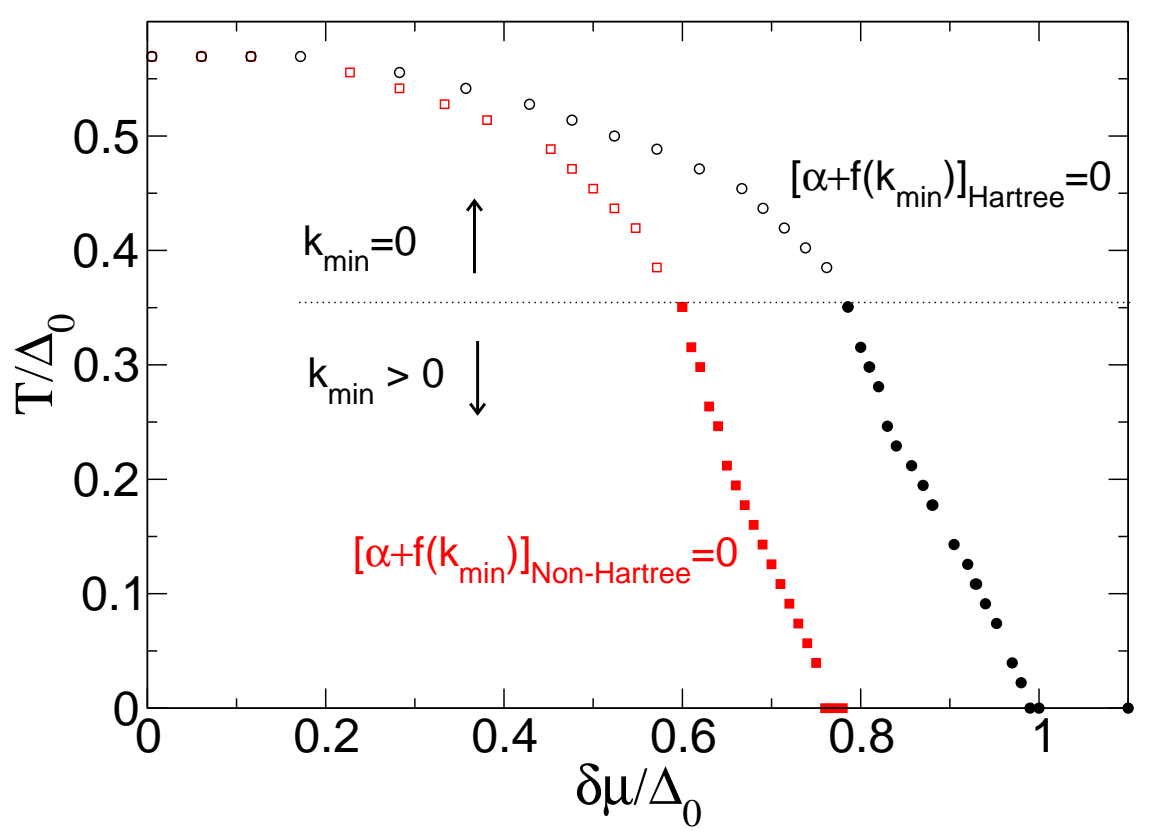

FIG. 4: (colored online) We show the curve marking a second order phase transition between normal and inhomogeneous superfluid phases in $\delta \mu, T$ space, for $g=-0.72$. In the curve marked by circles (black online), we include the Hartree corrections while the curve marked by squares (red online) does not include these. Where the symbols are hollow, the value of $k$ at which $\alpha+f(k)$ becomes zero, is zero, meaning that the instability is towards the formation of homogeneous condensates. It is reasonable that at smaller $\delta \mu$ and larger temperatures, the formation of homogeneous condensates is favored because the Fermi surface is smeared out and it is no longer advantageous to form Cooper pairs with non-zero net momenta.

If $k_{\min } \neq 0$, this point symbolizes the onset of the instability towards the formation of an inhomogeneous condensate. At lower $\delta \mu$, more momentum modes may become unstable. If the transition from normal to inhomogeneous superfluidity is actually first order, then we expect it to occur for values of $\delta \mu$ larger than the value we find using this second order analysis.

Fig. (44) shows the curve in $T, \delta \mu$ space, which tells us the value of $\delta \mu$ where the coefficient $\alpha+f(k)$ becomes zero for some $k$, as we decrease $\delta \mu$ from a large value keeping $T$ constant. Since we are looking at a second order phase transition line, once we take into account the shift in the chemical potentials due to the Hartree corrections, the results are consistent with the well known results for weak coupling. For example, at $T=0$, the value of $\delta \tilde{\mu}=\delta \mu-(-4 \pi a / m) \delta n$ at the phase transition is given by $\delta \tilde{\mu} / \Delta_{0} \sim 0.75$. Weak coupling Ginzburg-Landau calculations tell us that the transition should occur at $\delta \mu^{*}=0.754 \Delta_{0}$ with the most unstable momentum $k_{\min }$ given by $v_{F} k_{\min } / 2=1.2 \delta \mu^{*}$. We find that the value of $k$ which becomes unstable at $\delta \tilde{\mu} / \Delta_{0} \sim 0.75$ satisfies $v_{F} k_{\min } / 2 \sim 1.24 \delta \tilde{\mu}$. For reference we also show the LOFF boundary excluding the Hartree corrections using square dots (red online). The instability towards inhomogeneous condensates at $T=0$ develops at $\delta \mu / \Delta_{0} \sim 0.75$, and the value of $v_{F} k_{\min } / 2$ we find at this point is $1.37 \delta \mu$.

\section{CONCLUSIONS}

We calculate the effect of Hartree corrections on the phase transition curve separating the normal from the superfluid phase in the $T, \delta \mu$ plane, in the BCS regime. Our analysis is similar in spirit to the calculations 


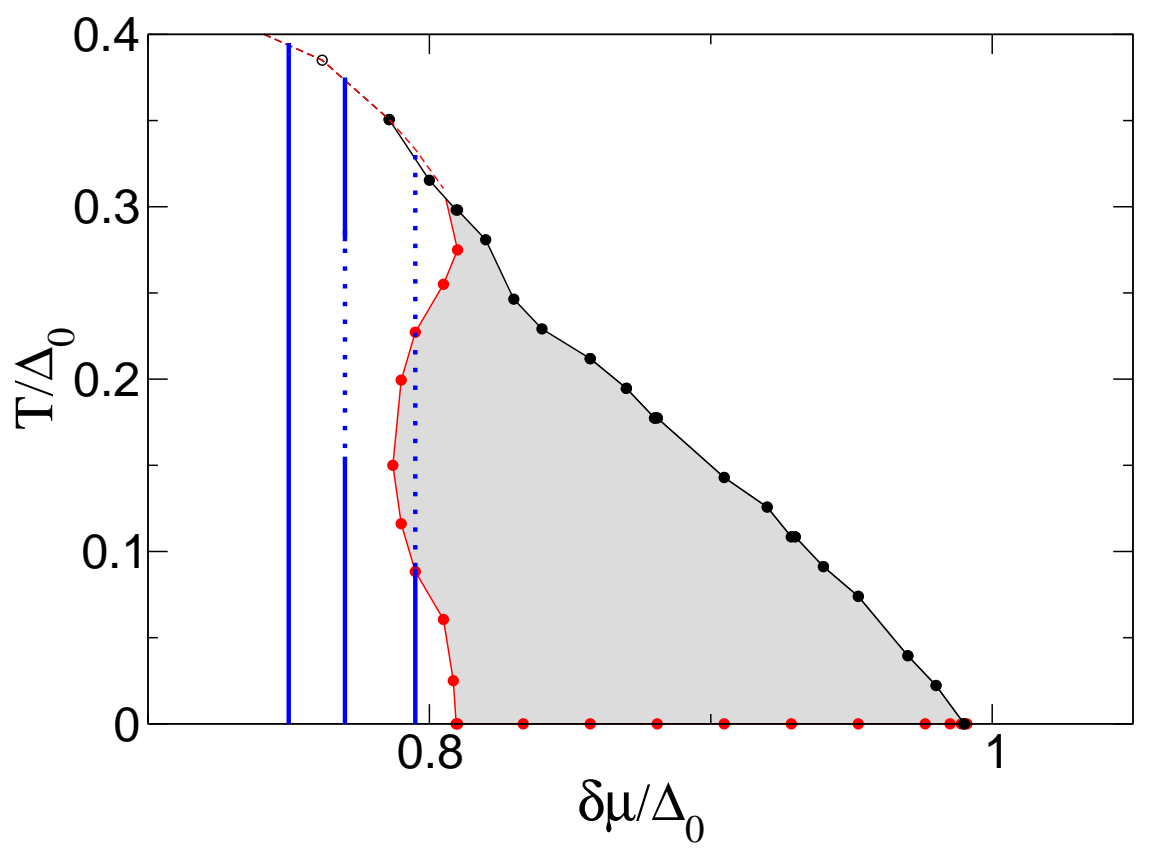

FIG. 5: (colored online) We show the phase boundaries between the normal and the inhomogeneous superfluid phases and the normal and the homogeneous superfluid phase, for $g=-0.72$. The lower curve (red online) represents the homogeneous superfluid boundary, while the upper curve (black online) marks the instability towards inhomogeneity. The two curves coincide for $\delta \mu \lesssim 0.77 \Delta_{0}$. The shaded region is where LOFF-like phases may exist. The three parallel thick lines (blue online) placed at $\delta \mu / \Delta_{0}=0.750,0.770$ and 0.795 , show the stability of the mean field background solution to the growth of inhomogeneities. In the temperature range for which a line is solid, the homogeneous condensate is stable, while for the temperatures for which a line is dotted, the condensate is unstable. The input for these lines comes from the data shown in Fig. (7).

performed by the authors of [14], who however concentrate on the unitary regime. In their work, the Hartree corrections are taken to be of a well motivated form [31] chosen so as to reproduce the data from Monte Carlo simulations performed at unitarity [7]. We work in the BCS regime, where the Hartree corrections can be calculated from first principles, as described above. Furthermore, we consider the instability of the homogeneous states with respect to the formation of inhomogeneous condensates.

The results are shown in Figs. (10 4) for $g=-0.72$. In figure Fig. (5), we zoom in on the large $\delta \mu$ region and show the homogeneous-normal and inhomogeneous-normal phase boundaries on the same diagram. The curve that is non-zero up to $\delta \mu \sim 0.99 \Delta_{0}$ (black online) shows the parameter values for which the coefficient of the $\Delta(\mathbf{k})^{2}$ term, in a Ginzburg-Landau expansion about the normal phase, becomes negative for some value of $k=k_{\mathrm{min}}$. This signals an instability towards the formation of an inhomogeneous condensate. The curve that meets the $x$-axis at $\delta \mu \sim 0.81 \Delta_{0}$ (red online) represents the points where the free energies of the homogeneous superfluid and the normal phase are equal. Therefore, the region between the boundaries, highlighted by a shading (gray online), is the region where we expect LOFF-like phases to exist. Our first conclusion is that at weak coupling, Hartree corrections do not destroy the parameter space where LOFF-like phases may exist.

Secondly, we find that the shape of the curve marking the transition from the homogeneous superfluid phase to the normal phase, is qualitatively altered (Fig. (1)). For a narrow range of $\delta \mu$ near the first order transition, we encounter three normal-superfluid transitions as we increase the temperature keeping $\delta \mu$ constant. There is a first order phase transition from the superfluid to the normal phase at a low temperature. As we increase the temperature, a "fragile" superfluid phase, where pairing is assisted by a 
temperature induced smearing of the Fermi surfaces, reappears in a window of temperatures.

As we shall see below, analysis of fluctuations about the mean field will tell us that this "fragile" superfluid may give way to an inhomogeneous phase (Figs. (5) 7)). For $\delta \mu / \Delta_{0}=0.750,0.770$ and 0.795 , the temperatures for which the homogeneous condensate is unstable is shown by the dotted lines (blue online) in Fig. (5).

If this mean field picture is taken as it is (and ignoring the inhomogeneous phases for a moment), this structure of the phase diagram could be observed in cold atomic traps. As we go away from the center of the trap, the effective chemical potential and therefore $\Delta_{0}$ decreases. Since $T$ and $\delta \mu$ are constant across the trap, this implies that $T / \Delta_{0}$ and $\delta \mu / \Delta_{0}$ increases. The "fragile" superfluid region can be probed by tuning the parameters, namely the number of the two species in the trap $N_{1}$ and $N_{2}$, the scattering length $a$, and the temperature $T$, so that for a given trap geometry, we pass through the "fragile" superfluid region as we go from the center of the trap to the outside.

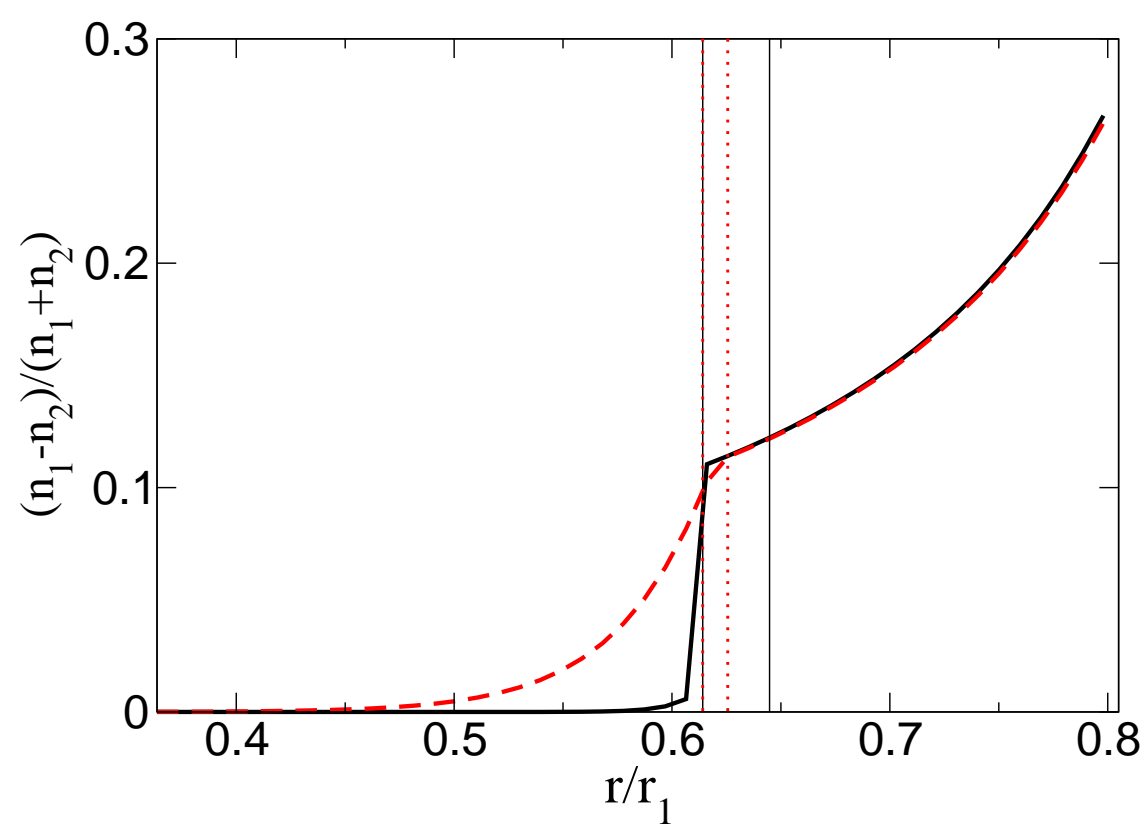

FIG. 6: (colored online) Polarization as a function of the $r$ plotted in units of $r_{1}$. The bold line (black online) corresponds to $T=0.1 \delta \mu$ and the dashed line (red online) to $T=0.36 \delta \mu$. Also shown are regions where the system is unstable to the growth of inhomogeneities. The thin continuous lines (black online) specify this region for $T=0.1 \delta \mu$ and the thin dashed lines (red online) mark this region for $T=0.36 \delta \mu$. The lower boundary marked by a thin dotted line, coincides with the left boundary of the thin continuous line. The upper boundary, however, appears at a larger $r$ for the continuous line, telling us that the window of inhomogeneous pairing is wider at lower temperature. For $T=0.1 \delta \mu$ the total value of $N$ in the trap is $3.21 \times 10^{(+7)}$ and the value of $\delta N$ is $1.26 \times 10^{(+6)}$. For $T=0.36 \delta \mu$ the total value of $N=3.20 \times 10^{(+7)}$ and the value of $\delta N=1.53 \times 10^{(+6)}$.

In Fig. (6) , we plot profiles of the polarization $p=\left(n_{1}-n_{2}\right) /\left(n_{1}+n_{2}\right)$ as a function of $r$, where $r$ is the distance from the center of the trap and $n_{1}$ and $n_{2}$ are the number densities of the two species. The trap is taken to be spherically symmetric, with a harmonic trapping angular frequency $\omega=100 \mathrm{rads} / \mathrm{s}, 9$, 10, 11, 12, 13]. The harmonic potential can be written as $V(r)=1 / 2 \times \hbar \omega \times\left(r / r_{0}\right)^{2}$ with $r_{0}=\sqrt{ }(\hbar /(m \omega))$, where $m$ is the mass of ${ }^{6} \mathrm{Li}$ atoms. In natural units, $\omega=2.599 \times 10^{(-10)}(\mathrm{eV}), m=5.61 \times 10^{(+9)}(\mathrm{eV})$ and $r_{0}=0.82816(\mathrm{eV})^{-1}$. 
With the geometry of the trap specified, we now try to tune the parameters of the experiment $N=$ $\left(N_{1}+N_{2}\right) / 2, \delta N=\left(N_{1}-N_{2}\right) / 2, a$ and $T$ and try to identify the region where there is a phase transition from the superfluid phase to the unpaired phase. This is interesting because of two reasons. Firstly, since the polarization rises rapidly at this phase transition boundary, this boundary can be recognized in the trap by observing the polarization as a function of $r$. Secondly, the inhomogeneous phases are likely to be found near this boundary at low temperatures, and we would like to estimate the range of $r$ where these phases are likely to exist. We choose $a=-0.0205(\mathrm{eV})^{-1}$, for which the atomic system, especially at the center of the trap, can not be considered weakly coupled, and hence our approximations may not be quantitatively accurate near the center of the trap. But the large coupling amplifies the features in the polarization profile that we are looking for. We use these features to deduce qualitative conclusions which may be more general than our approximations.

Although the true experimental variables are $N$ and $\delta N$, we find it more convenient to fix the values of the average chemical potential at the center of the trap, $\mu_{0}$, and the chemical potential splitting $\delta \mu$, and calculate $N$ and $\delta N$ in terms of these. The average chemical potential at a point $r$ in the trap is $\mu(r)=\mu_{0}-V(r)$. Plotted in Fig. (6) is $p$ as a function of $r$ for $\mu_{0}=1.314 \times 10^{(-7)}(\mathrm{eV})$ and $\delta \mu=1.2 \times 10^{(-8)}$ (eV) for two different temperatures, $T=0.1 \delta \mu$ and $T=0.36 \delta \mu$. We focus on the region near the place where we observe the jump in the value of the polarization. The radius $r$ has been plotted in units of $r_{1}$ defined as the radius where the effective chemical potential of $\psi_{1}$ species is zero, i.e. $r_{1}=r_{0} \sqrt{ }\left(2\left(\mu_{0}+\delta \mu\right) / \omega\right)=33.22 r_{0}$.

We see that, as expected, the jump in the polarization at the phase transition boundary becomes less sharp as we increase the temperature. We also mark the region where the system is unstable to the formation of inhomogeneous phases. We also see that the range of $r$ where inhomogeneity may develop, decreases in size as we increase the temperature.

But we expect that this mean field picture will be modified due to quantum corrections. One thing to note from the double-dot-dashed curve (red online) in Fig. (2) is that the free energy curve as a function of $\Delta$ is very shallow. The mass of the fluctuations in the magnitude of the condensate is therefore very small and therefore we expect quantum corrections to be important in this "fragile" superfluid state.

The role of quantum corrections can be understood by considering the effective action describing the fluctuations of the two fermion condensate about the mean field value. If the system is stable to small fluctuations of the condensate, we may calculate their contribution to the free energy and see if they increase the mean field value of the free energy or decrease it. Recently, the effect of fluctuations on the free energy function has been calculated for $T=0$ and $\delta \mu=0$ [32]. These calculations suggest that the mean field calculations overestimate the value of $\Delta_{0}$ as well as the value of $n$ in the superfluid state.

The first step in such a calculation is to find the effective potential describing the dynamics of the fluctuation field and seeing if the system is stable to small fluctuations in the mean field value. In the following, we analyze the stability of the "fragile" superfluid to fluctuations that depend on position and are purely imaginary for real $\Delta$. I.e., we consider a condensate of form

$$
\left\langle\psi_{\alpha}(\mathbf{r}) \psi_{\beta}(\mathbf{r})\right\rangle=\frac{1}{\lambda} \epsilon_{\alpha \beta}(\Delta+i \eta(\mathbf{r})),
$$

where $\Delta$ and $\eta$ are both real. The reason for considering purely imaginary fluctuations (eq. (34)) is that it is clear from the free energy curve at $T=0.24 \Delta_{0}$ in Fig. (2) that the free energy is at a local minimum at the mean field value $\Delta$, meaning that the system is stable to spatially uniform variation in the magnitude of the condensate. One could potentially consider position dependent fluctuations in the magnitude of the condensate but we only consider real $\eta(\mathbf{r})$ in eq. (34), which is related to position dependent fluctuations in the phase of the condensate. The effective potential describing the dynamics of $\eta$ can be found in a Ginzburg-Landau expansion in the same way as was done in section [IIB. where we considered inhomogeneous fluctuations about the normal phase. The only difference now is that the background has a non-zero 


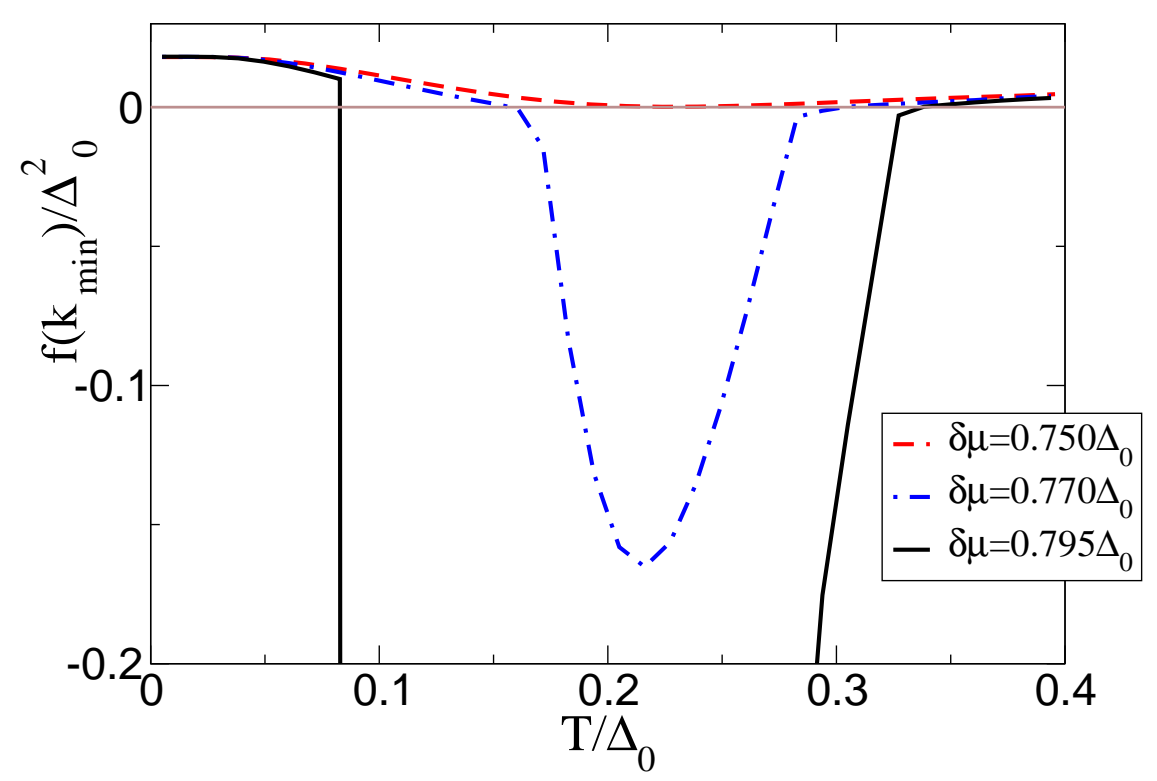

FIG. 7: $f_{\min }$ as a function of $T$ at three values of $\delta \mu, \delta \mu=0.750 \Delta_{0}, \delta \mu=0.770 \Delta_{0}$ and $\delta \mu=0.795 \Delta_{0}$. We take $g=-0.72$ as before. Looking first at $\delta \mu=0.795 \Delta_{0}, f_{\min }$ is clearly negative in the unpaired region, corresponding to $T / \Delta_{0} \in(0.08,0.22)$. It is also negative in the "fragile" superfluid region lying in $T / \Delta_{0} \in(0.22,0.33)$. Now considering $\delta \mu=0.770 \Delta_{0}$, we find that the homogeneous condensate is unstable in $T / \Delta_{0} \in(0.16,0.29)$. Finally for $\delta \mu=0.750 \Delta_{0}$ (and lower), we find that the homogeneous condensate is stable for all $T$. These three curves form the basis of the thick dashed lines (blue online) in Fig. (5).

condensate value, $\Delta$. The final answer has the form,

$$
\int d^{3} \mathbf{r}\left(\Omega(\Delta(\mathbf{r}))-\Omega_{N}\right)=\int \vec{d}^{3} \mathbf{k} \eta(\mathbf{k}) \eta(-\mathbf{k})\left\{f_{\Delta}(|\mathbf{k}|)\right\}
$$

with,

$$
f_{\Delta}(|\mathbf{k}|)=\frac{1}{4} \int \pi^{3} \mathbf{p}\left\{\frac{(\tilde{\xi}(\mathbf{p}+\mathbf{k})-\tilde{\xi}(\mathbf{p}))^{2}}{\left(\tilde{\epsilon}(\mathbf{p}+\mathbf{k})^{2}-\tilde{\epsilon}(\mathbf{p})^{2}\right)}\left[\frac{g(\tilde{\epsilon}(\mathbf{p}))}{\tilde{\epsilon}(\mathbf{p})}-\frac{g(\tilde{\epsilon}(\mathbf{p}+\mathbf{k}))}{\tilde{\epsilon}(\mathbf{p}+\mathbf{k})}\right]\right\}
$$

The form in eq. (35) closely resembles the form in eq. (30), except now $\alpha=0$, which can be easily understood as the consequence of the fact that a position independent fluctuation in the phase of the condensate can not change the free energy.

In Fig. (7) we plot the minimum value of $f_{\Delta}(k)$ (minimized over $k=|\mathbf{k}|$ ) as a function of $T$ at $\delta \mu / \Delta_{0}=$ $0.795,0.770$ and 0.750 for $g=-0.72$. For $\delta \mu / \Delta_{0}=0.795$, we see that the minimum value, $f\left(k_{\min }\right)$, is negative in the "fragile" superfluid region lying in $T / \Delta_{0} \in(0.22,0.33)$, indicating that it is unstable with respect to developing a position dependent phase modulation. We see (Fig. (77) that as we decrease $\delta \mu$, the homogeneous superfluid region becomes stable for all $T$, as it should.

In light of this instability of the "fragile" superfluid, our results of the polarization profiles for the atomic trap may need to be modified. To find the correct profile, we will need to calculate the number densities of the two species in an inhomogeneous condensate more accurately than we have done above. We leave this calculation for future work. Another issue that warrants further investigation is the role of polarization effects (also known as the Gorkov-Barkhudarov corrections) that can reduce the gap. In weak coupling, medium polarization results in screening the interactions and reduces the gap and the critical temperature by a factor of $(4 e)^{(1 / 3)} \sim 2.2$ when $\delta \mu=0[20,21]$. If we were to incorporate this reduction by modifying the 
four-fermion coupling in the pairing channel to mimic the Gorkov suppression at $T=0$ and $\delta \mu=0$, it is easy to argue that the phase boundary (shown in Fig. 1) would simply be scaled by the same reduction factor 2.2 both in temperature and $\delta \mu$. However, a systematic calculation of these medium effects at moderate coupling is challenging and preliminary investigations suggest that the gap is not as strongly suppressed [33]. Further, even in weak coupling the nature of this suppression is not understood at finite population imbalance. We leave a systematic calculation of how Gorkov corrections evolve with coupling strength and $\delta \mu$ for future work.

Despite these aforementioned caveats, we reiterate that our investigation here points to several interesting qualitative features that arise only when Hartree corrections are included. In particular we see that the region in phase diagram susceptible to gradient instabilities is moderately enhanced by these corrections. The shape of the normal-superfluid phase boundary is shown to depend on the nature of these mean field energy shifts since they differ in the two phases in the vicinity of the first-order transition. In particular the Clogston-Chandrashekar point, which is given by $\delta \mu=\Delta / \sqrt{2}$, is shifted to higher values of $\delta \mu$ by these corrections.

\section{ACKNOWLEDGMENTS}

RS thanks Krishna Rajagopal for discussion and suggestions. The authors also acknowledge discussions with Michael Forbes, Joe Carlson, Mark Alford and Alex Gezerlis. This research was supported by the Dept. of Energy under contract W-7405-ENG-36 and by the LANL/LDRD Program.

[1] J. Bardeen, L. N. Cooper, and J. R. Schrieffer, Phys. Rev. 108, 1175 (1957).

[2] P. Fulde and R. A. Ferrell, Phys. Rev. 135, A550 (1964).

[3] A. Larkin and Y. Ovchinnikov, Sov. Phys. JETP 20, 762 (1965).

[4] M. M. Forbes, E. Gubankova, W. V. Liu, and F. Wilczek, Physical Review Letters 94, 017001 (pages 4) (2005), URL http://link.aps.org/abstract/PRL/v94/e017001

[5] H. Muther and A. Sedrakian, Physical Review Letters 88, 252503 (2002), URL http://xxx.lanl.gov/abs/cond-mat/0202409

[6] A. Sedrakian, J. Mur-Petit, A. Polls, and H. Muther, Phys. Rev. A 72, 013613 (2005), URL http://xxx.lanl.gov/abs/cond-mat/0504511

[7] J. Carlson and S. Reddy, Phys. Rev. Lett. 95, 060401 (pages 4) (2005), URL http://link.aps.org/abstract/PRL/v95/e060401

[8] D. T. Son and M. A. Stephanov, Phys. Rev. A 74, 013614 (2006), URL http://xxx.lanl.gov/abs/cond-mat/0507586.
G. B. Partridge, W. Li, R. I. Kamar, Y. an Liao, and R. G. Hulet, Science 311, 503 (2006), URL

[9] G. B. Partridge, W. Li, R. I. Kamar, Y. an Liao, and R. G. Hulet, Science 311, 503 (2006), URL
http://xxx.lanl.gov/abs/cond-mat/0511752.

10] G. B. Partridge, W. Li, Y. A. Liao, R. G. Hulet, M. Haque, and H. T. C. Stoof, Phys. Rev. Lett. 97, 190407 (2006), URL http://xxx.lanl.gov/abs/cond-mat/0608455

[11] Y. Shin, C. H. Schunck, A. Schirotzek, and W. Ketterle, Phys. Rev. Lett. 99, 090403 (pages 4) (2007), URL http://link.aps.org/abstract/PRL/v99/e090403

[12] Y. Shin, M. W. Zwierlein, C. H. Schunck, A. Schirotzek, and W. Ketterle, Phys. Rev. Lett. 97, 030401 (2006), URL http://xxx.lanl.gov/abs/cond-mat/0606432.

[13] Y.-I. Shin, C. H. Schunck, A. Schirotzek, and W. Ketterle (2007), URL http://xxx.lanl.gov/abs/0709.3027.

[14] K. B. Gubbels, M. W. J. Romans, and H. T. C. Stoof, Physical Review Letters 97, 210402 (2006), URL http://xxx.lanl.gov/abs/cond-mat/0606330.

[15] M. M. Parish, F. M. Marchetti, A. Lamacraft, and B. D. Simons, Nature 3, 124 (2007), URL http://www .nature.com/nphys/journal/v3/n2/abs/nphys520.html

[16] H. Burkhardt and D. Rainer, Annalen der Physik 506, 181 (1994), URL http://adsabs.harvard.edu/abs/1994AnP...506..181B. 
[17] A. I. Buzdin and H. Kachkachi, Physics Letters, Section A: General, Atomic and Solid State Physics 225 (4-6), 341 (1997).

[18] R. Combescot and C. Mora, Europhysics Letters $\quad 68, \quad 79 \quad$ (2004), URL http://xxx.lanl.gov/abs/cond-mat/0311042.

[19] G. Sarma, Phys. Chem. Solids 24, 1029 (1963).

[20] L. Gorkov and T. Melik-Barkhudarov, Sov. Phys. JETP 13, 1018 (1961).

[21] H. Heiselberg, C. J. Pethick, H. Smith, and L. Viverit, Physical Review Letters 85, 2418 (2000), URL http://xxx.lanl.gov/abs/cond-mat/0004360

$[22]$ H. Abuki and T. Kunihiro, Nuclear $\quad$ Physics A $\quad 768, \quad 118 \quad$ (2006), URL http://xxx.lanl.gov/abs/hep-ph/0509172.

[23] D. Blaschke, S. Fredriksson, H. Grigorian, A. M. "Oztas, and F. Sandin, Physical Review D 72, 065020 (2005), URL http://xxx.lanl.gov/abs/hep-ph/0503194.

[24] K. Fukushima, C. Kouvaris, and K. Rajagopal, Physical Review D 71, 034002 (2005), URL http://xxx.lanl.gov/abs/hep-ph/0408322

[25] M. Huang and I. Shovkovy, Nuclear $\quad$ Physics $\quad$ A $\quad 729, \quad 835 \quad$ (2003), URL http://xxx.lanl.gov/abs/hep-ph/0307273.

[26] S. B. Ruester, V. Werth, M. Buballa, I. A. Shovkovy, and D. H. Rischke, Physical Review D 73, 034025 (2006), URL http://xxx.lanl.gov/abs/hep-ph/0509073.

[27] I. Shovkovy and M. Huang, Physics Letters B $564, \quad 205 \quad$ (2003), URL http://xxx.lanl.gov/abs/hep-ph/0302142.

[28] H. J. Warringa (2006), URL http://xxx.lanl.gov/abs/hep-ph/0606063.

[29] A. Sedrakian and U. Lombardo, Physical Review Letters 84, $602 \quad$ (2000), URL http://xxx.lanl.gov/abs/nucl-th/9907076

[30] E. Gubankova, M. Mannarelli, and R. Sharma (2008), URL http://xxx.lanl.gov/abs/0804.0782.

[31] B. M. Fregoso and G. Baym, Physical Review A 73, 4 (2006), URL http://xxx.lanl.gov/abs/cond-mat/0602191.

[32] B. R. Diener, R. Sensarma, and M. Randeria (2007), URL http://xxx.lanl.gov/abs/0709.2653.

[33] H.-J. Schulze, A. Polls, and A. Ramos, Phys. Rev. C 63, 044310 (2001). 